\title{
Mujeres en el Legislativo Federal, una Mirada Espacial México 1988-2011
}

\author{
Women in the Federal Legislature, a Look on Spatial \\ Mexico 1988-2011
}

\author{
Verónica Ibarra García \\ Universidad Abierta y Educación a Distancia UNAM \\ maveroibar@gmail.com
}

Angélica Lucía Damián Bernal

Universidad Nacional Autónoma de México

lucia.damian@epadeq.com.mx

\section{Resumen}

El artículo muestra el proceso de incorporación de las mujeres en el poder legislativo Federal en México, tanto en la Cámara de Diputados como de Senadores, muestra como a lo largo del tiempo el proceso ha sido paulatino, gradual y de difícil acceso para las mujeres. Se elaboraron cifras porcentuales para identificar las diferencias espaciales, por entidad federativa de 1988 a 2011, cuyos resultados encontrados fueron georeferenciados para analizarlos con una perspectiva espacial, detallando en cuáles entidades la presencia de mujeres es constante, nula $\mathrm{u}$ ocasional. Al final se presenta una evaluación de la situación.

Palabras clave: Mujeres, Poder, Georeferencia, México.

\begin{abstract}
The article shows the incorporating process of women in the Federal legislature in Mexico, both in the Chamber of Deputies and Senate, shows how, along the time, the process has been progressive, gradual and difficult to access for women. Percentage figures were developed to identify spatial differences, by state, from 1988 to 2011, whose results were georeferenced to analyze a spatial perspective, detailing in which entities the presence of women has been constant, null or occasional. At the end, it provides an assessment of the situation.
\end{abstract}

Keywords: Women; Power; Georeference; Mexico. 


\section{Mujeres y poder en el tiempo-espacio de México}

La demanda del reconocimiento formal a la participación política de las mujeres ha sido documentada por diversos estudios, el movimiento feminista en México es identificado de manera paralela al establecimiento del régimen posrevolucionario, aunque tiene sus antecedentes desde el siglo XIX en México, como en el resto del mundo.

Las mujeres mexicanas tuvieron una destacada participación en el nuevo régimen posrevolucionario, a su vez demandaban la formalización de las mismas en las estructuras de poder, tal es el caso de Hermila Galindo, quien fue una destaca luchadora social y política que pugnaba por el derecho al voto, en la década de los veinte, del siglo pasado, desde su cercanía como secretaria particular del presidente Venustiano Carranza y quien fue la primera mujer en registrar una candidatura al congreso, cuando aún las mujeres no podían acceder al mismo.

Ahora bien mientras que en la escala federal el acceso de las mujeres a las estructuras de poder fue una larga serie de negociaciones con altibajos que dio resultados hasta el 17 de octubre de 1953, en la escala estatal hubo verdaderos avances en la provincia mexicana, tal es el caso de Yucatán en el Sureste Mexicano, entidad en donde se organizó el primer encuentro de feministas en 1917 bajo el auspicio del general sinaloense, Salvador Alvarado, entonces gobernador del estado de Yucatán, además en esta misma entidad se reconoció el acceso de las mujeres a las estructuras de poder municipal en las elecciones de 1922, posteriormente los estados que lograron el reconocimiento de las mujeres fueron Chiapas, Tabasco y San Luis Potosí en 1925. Pero fue hasta 1946 cuando el entonces presidente Miguel Alemán Valdés reconoció el derecho a las mujeres a ser electas presidentas municipales, para todo el territorio nacional.

Las tres primeras mujeres que 'accedieron' en la cámara de Senadores fueron en calidad de suplentes, por las entidades de Aguascalientes, Chiapas y Nuevo León, en 1958.

Para que México tuviera su primera gobernadora pasaron doce años más, en 1970 Griselda Álvarez gobernó Colima por seis años, en 1987 Beatriz Paredes fue la segunda gobernadora del país por el estado de Tlaxcala. La década de los noventa inició con la gobernadora suplente en Chihuahua, Martha Lara Alatorre, también en el estado de Yucatán llego una suplente al palacio de Gobierno, en este caso Dulce
María Sauri Riancho (1991-1994) la década concluyó con una tercera suplente, Rosario Robles en la capital del país en donde gobernó de 1999 al año 2000.

El siguiente siglo dio paso a la llegada de dos gobernadoras que ganaron la elección, en Zacatecas Amalia García del 2004 al 2010 y Yucatán, en donde Yvonne Ortega Pacheco llego el $1^{\circ}$ de agosto de 2007 (MEDINA, 2010).

Así podemos observar como a menor grado de poder político mayor facilidad de las mujeres en acceder a él, ya que primero se logró participar en las presidencias municipales, posteriormente a las diputaciones federales, después las mujeres pudieron llegar a ser Senadoras. Ello también ha generado que prevalezcan los estudios de las mujeres en su acceso al poder político local, de las presidencias municipales y diputadas locales, como lo demuestran las investigaciones sobre presidentas municipales (MASSOLO, 1995; BARRERA-MASSOLO, 1998), de las ediles por los diferentes partidos políticos (BARRERA 2000; 2001; 2002), de las Priístas (HIDALGO, 2000), de las Panistas (RODRIGUEZ, 2000; MARTÍNEZ, 2002, LIMON AGUIRRE, 2002) y de las Perredistas (GONZÁLEZ, 2002).

Ahora bien, en cuanto a las mujeres en el poder no fue un proceso que se haya planteado desde el régimen político mexicano sino que fue el movimiento feminista sostenido, quienes no dejaron de luchar por el derecho a elegir y ser electas. Ya que si bien la Constitución Mexicana no impedía la participación política de las mujeres tampoco era explícita su posición con respecto al género femenino. Una vez logrado el reconocimiento político a las mujeres con derecho a elegir y ser elegidas inicio un lento proceso de incorporación de las mismas al poder.

Es pertinente considerar que México tiene una régimen legislativo mixto, es decir de mayoría absoluta y mayoría relativa, este último fue como respuesta a la falta de legitimidad que presentaba el partido hegemónico en la década de los setenta, a partir de las reformas de 1977 establecida por Jesús Reyes Heroles, ante lo que se dictaminó el principio de representación proporcional, en la Cámara de Diputados o Baja, mismo principio que se implementó en 1994 para la Cámara de Senadores o Alta, para la primera existe un total de 500 Diputados, 300 de mayoría absoluta y 200 de representación proporcional, en listas por circunscripción electoral, mientras que para la segunda hasta las elecciones de 1991 había 64 Senadores, dos por entidad federativa, elegidos por el principio de mayoría absoluta, pero a partir de 1994 ésta se incrementó a 128, tres elegidos por mayoría absoluta y un cuarto por representación proporcional en una sola lista nacional. 
Para poder identificar a las mujeres en el poder legislativo por entidad federativa se tomaron los resultados dados por las legisladoras de mayoría absoluta, y para las de mayoría relativa se tomó como referencia el estado en donde las mismas han desarrollado su carrera política, es decir es una combinación de elementos cuantitativos con cualitativos, en este caso esto fue definido así porque son claramente identificadas por su trabajo político en un lugar específico, al mismo tiempo que dichas políticas se convierten en referentes para otras mujeres.

Ahora bien aquí analizamos la representación que han logrado las mujeres en el Legislativo Federal, tanto Diputadas como Senadoras. Para el caso de diputadas y para poder comparar entre entidades federativas hemos trabajado con porcentajes, con ello establecimos rangos cada 20 unidades, Muy bajo menor a 20, Bajo 21 a 40, Medio 41 a 60, Alto 61 a 80 y Muy alto mayor a 80 , mismo que quedan plasmados en los mapas, mismos que permiten tener una visión espacial de las legisladoras.

Número de Legisladoras por entidad X100 Número total de legisladores (mujeres y hombres)

Para el caso de las Senadoras, cada entidad vota por tres senadoras y de acuerdo a la proporción nacional por cada partido se establece, un cuarto legislador por representación proporcional, para este caso se realizó el mismo procedimiento que con el de diputadas con representación proporcional, se tomó como base la entidad en donde han desarrollado su carrera política.

\section{En la escala federal del periodo 1988-2012}

La gráfica 1, muestra la presencia absoluta $\mathrm{y}$ porcentual de legisladoras de 1988 a 2012, identificamos que el año con menor presencia de mujeres en el Legislativo Federal fue el de 1991-1994, tanto en la Cámara de Diputados como de Senadores, a partir de entonces predomina un constante acceso de mujeres en dicho poder. En la Cámara Baja, el punto de inflexión es evidente en el año 1991, mientras que para la Cámara Alta, se identifican tres momentos de

disminución 1991, 1997 y 2003 contrariamente a lo que se esperaría dado el discurso de género que se ha instalado en la clase política y a pesar del compromiso adquirido al haber firmado la plataforma de Beijín 1995, en el que acordó establecer cuotas de género, mismas que se han asentado jurídicamente en cláusulas en donde ningún género debe rebasar el 70 por ciento de representación, primer objetivo no cumplido. Máxime cuando el número de electoras rebasa el número de electores varones como se ha demostrado en el estudio realizado por De la Barra (2008).

\section{Cámara de Diputados}

Como ya se ha sostenido la cámara baja está integrada por 500 diputados, y es evidente la escasa participación política de las mujeres, nunca se ha llegado al 30\% que la Ley indica, con base en ello podemos establecer un déficit en la equidad de género, para el análisis comparativo entre las entidades federativas como ya se estableció fue con base en porcentajes.

Partimos entonces de la premisa de la baja participación de mujeres en la cámara de Diputados Federal, en términos generales, pero también identificamos algunas entidades con presencia constante del género femenino en un rango medio y ocasionalmente alto, en esta situación destaca el estado de Quintana Roo, que ha sobresalido en las legislaturas LIV, LV, LVII y LIX por tener una presencia media de mujeres; en la LVI alta y en la legislatura LX muy alta, 
si bien en dos legislaturas estuvo en rango bajo, siempre contó con diputadas y no obtuvo un nivel muy bajo, ésta es la entidad con un comportamiento mayormente sostenido en la participación de las mujeres.

Otros estados a destacar porque en alguna ocasión han tenido participación alta son, Campeche (LX legislatura 2006-2009) y Tlaxcala (LXI legislatura 2009-2012); Nayarit sobresale en la legislatura LXI, tiene una proporción muy alta, sin embargo, son estados que anteriormente no se destacan por su permanente y alto rango de incorporación de las mujeres en la cámara de Diputados Federal. Por lo que consideramos que la presencia de mujeres es aleatoria y no una constante que hable de una mayor apertura a la participación femenina o bien a una cultura política con perspectiva de género.

En este sentido sólo dos entidades muestran una presencia sostenida de mujeres en la diputación federal con representantes que por una ocasión alcanzaron el de una alta presencia, Quintana Roo y Nayarit.

Como podemos observar con este breve análisis, es aún muy escasa la presencia de mujeres en el legislativo federal, no alcanza el 30\% establecido según los acuerdos de Beijín 1995 y 'aplicable' a partir de 2002, sin embargo es evidente que esta cláusula tuvo una repercusión positiva, ya que tuvo un importante crecimiento del 16 al $23 \%$ en la LIX legislatura (2003-2006), como se puedo observar en el gráfico uno. Las entidades con una presencia sostenida de mujeres en el Legislativo son sólo tres de 32, D.F. Nayarit y Quintana Roo.

\section{Cámara de Senadoras}

Ahora bien en un análisis sobre la presencia de mujeres en la Cámara alta o de Senadores, para el mismo periodo de 1988 a 2012 se observa un primer periodo de inestabilidad ya que en 1988 existen diez Senadoras, pero tres años después son sólo cinco de 60 Senadores para la República es decir menos del 10\%. Posteriormente sube a 12 en la legislatura de 19941997, sin embargo aunque aumenta en términos absolutos, en términos relativos tiene una gran descenso ya que a partir de este año, aumenta el número de escaños para la cámara de Senadores al doble pasa de 64 a 128, por lo que para el sexenio de 1997-2000 continua con su tendencia a la baja y disminuye nuevamente a 9 senadoras. Sin duda fue este el peor sexenio (1994-2000) de las mujeres en el poder legislativo federal (senadoras y diputadas).

El siguiente periodo de 2000 al 2006 aumenta a 20, coincide con el impulso a la cuota de género que México signa en Beijín en 1995, mismo acuerdo que sería efectivo a partir del año 2002. Finalmente en el sexenio de 2006-2012, incrementó el número de Senadoras, las cifras oficiales identifican a treinta, sin embargo un análisis más exhaustivo, demuestra que sólo accedieron veintiocho en términos reales, y una más dejó su curul para competir por la gubernatura que gano, situación por demás extraña entre las políticas no así entre los políticos.

\section{Análisis espacial}

Al realizar un análisis espacial podemos observar que de 1988 a 1991, hubo diez senadoras de las siguientes entidades, Baja California, Colima, Chiapas, Distrito Federal, Hidalgo, Jalisco, Oaxaca, Quintana Roo, Tamaulipas y Yucatán. Sólo cuatro coincidieron en tener al mismo tiempo Senadoras y Diputadas, en el mejor rango (medio) estuvieron Quintana Roo y Colima, seguidas de Yucatán y Oaxaca. Tres entidades tuvieron presencia baja de diputadas Guerrero, Querétaro y Nayarit, pero ninguna Senadora. El resto de las entidades tienen una presencia muy baja de diputadas 25 de 32 y tampoco ninguna senadora. Sin duda era un panorama nada positivo. (ver mapa 1).

En el trienio de 1991-1994, hubo una drástica reducción de senadoras de diez disminuyeron a cinco, de ellas tres quedaron del periodo anterior; Baja California, Oaxaca y Yucatán, y dos más se incorporaron Nuevo León y Querétaro. También es evidente la disminución de mujeres en la Cámara de diputados, sólo tres entidades muestran rangos de media y baja. Veintinueve de treinta y dos entidades estaban en el rango de muy bajo. Este fue el trienio de menor acceso de las mujeres al poder legislativo, como se demuestra en el mapa 2.

Durante el trienio de 1994 al 1997, se recuperó la presencia de mujeres en la Cámara alta, fueron 12 las legisladoras que accedieron al Senado: Campeche, Colima, Chiapas, Chihuahua, Distrito Federal, Durango, Guerrero, Edo de México, Oaxaca, Querétaro, Tamaulipas y Tlaxcala. Mientras que dos senadoras quedaron del trienio anterior, Nuevo León y Querétaro. Por lo que hubo un total de 14. Al mismo tiempo se incrementó la presencia de diputadas en esta legislatura, Quintana Roo se presentó con el mayor rango, de alto, seguido de Colima con medio, y ocho entidades con rango bajo. Veintidós entidades se mantuvieron en el rango de muy bajo. (ver mapa 3 ) 
Mujeres en el Legislativo Federal, una Mirada Espacial

México 1988-2011
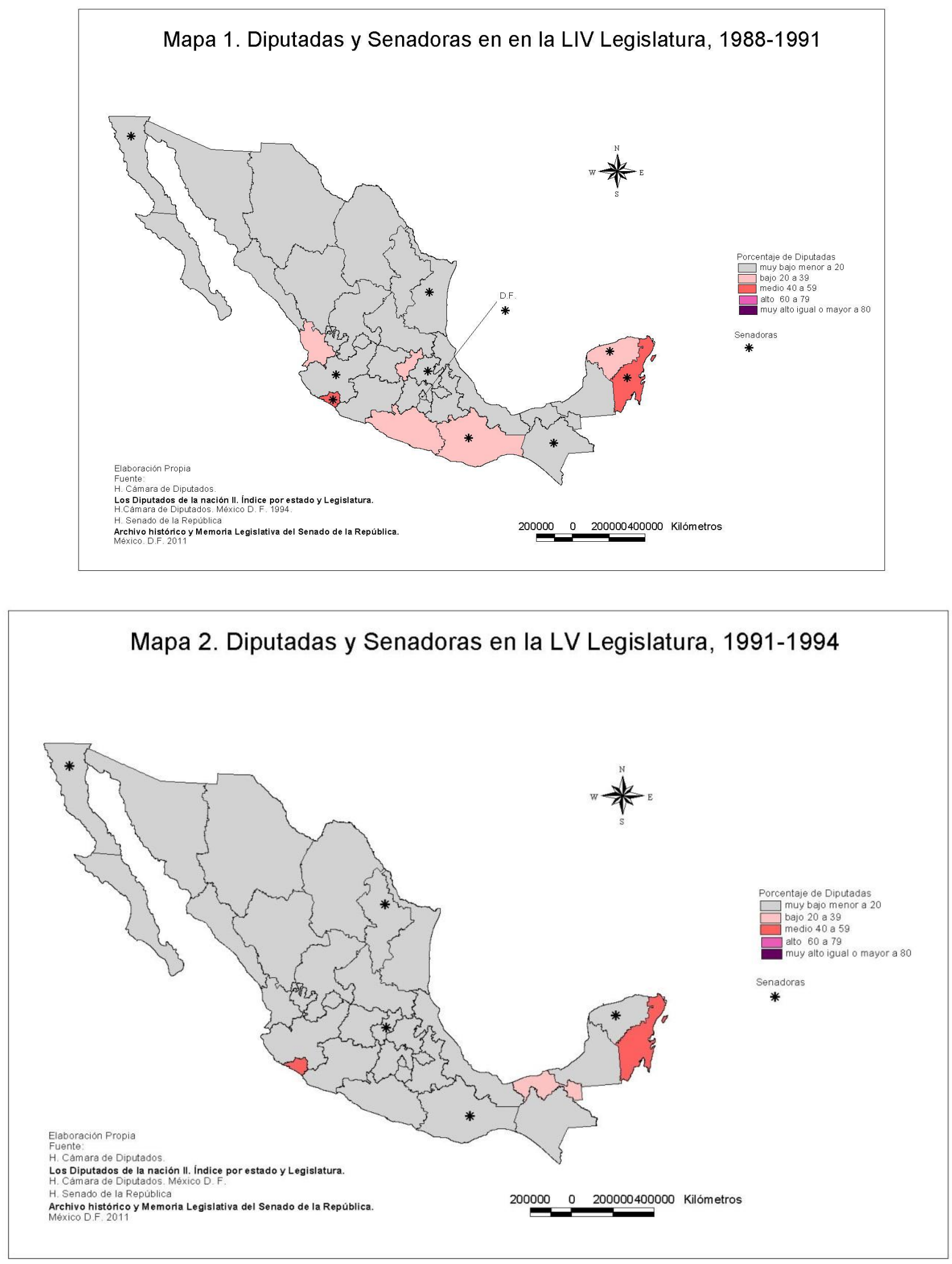

Verónica Ibarra García e Angélica Lucía Damián Bernal

Revista Latino-americana de Geografia e Gênero, Ponta Grossa, v. 4, n. 1, p. 2-13, jan. / jul. 2013. 


\section{Mapa 3. Diputadas y Senadoras en la LVI Legislatura, 1994-1997}

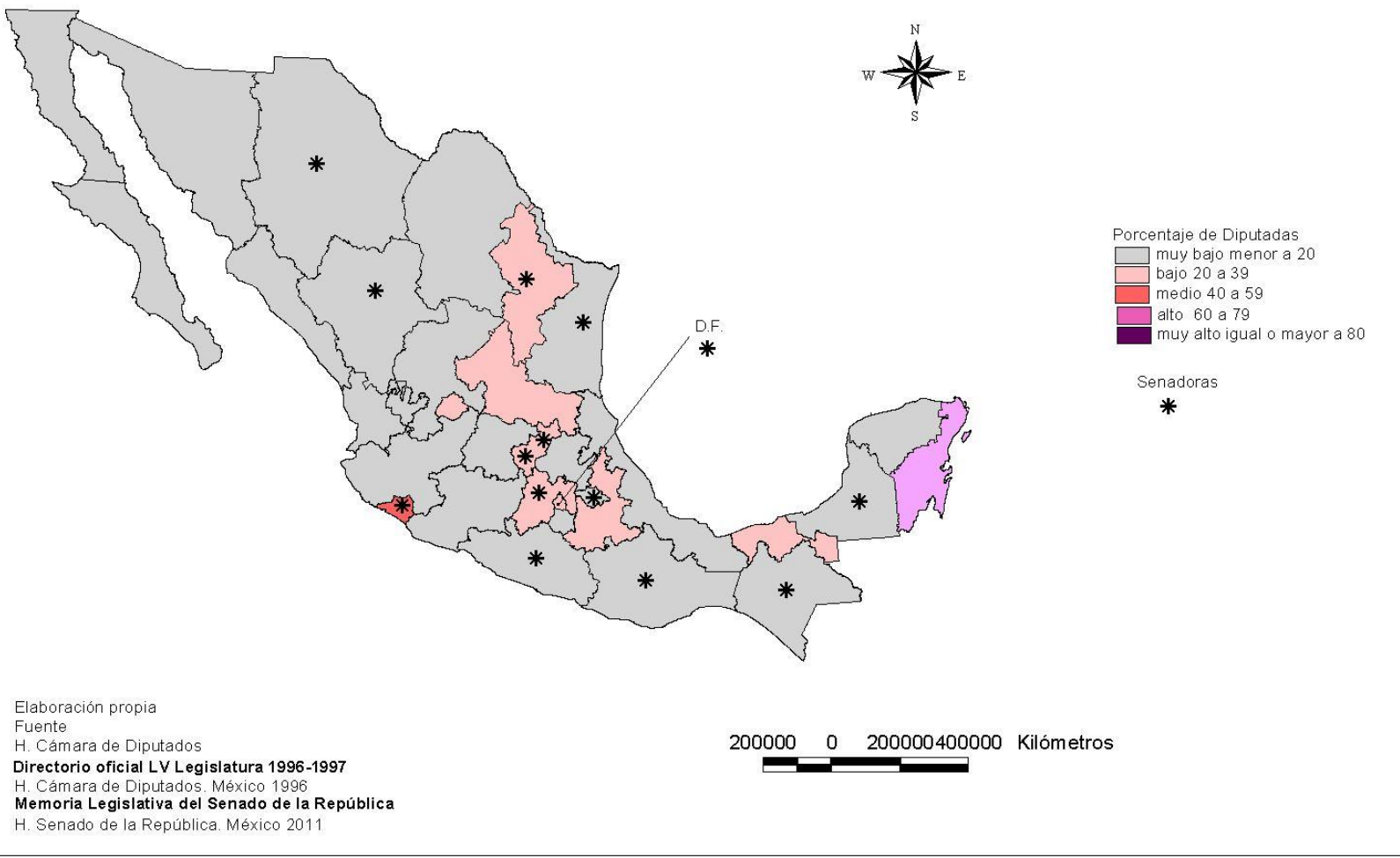

Colima es la entidad con mejor posicionamiento, tiene una presencia media de diputadas y cuenta con una senadora, tiene representantes en ambas cámaras. Le sigue Estado de México, Nuevo León, Querétaro y Distrito Federal donde se encuentran legisladoras en ambas cámaras, pero en el caso de diputadas se encuentran en el rango bajo. Quintana Roo aumenta, y de una legislatura a otra cambia de porcentaje de medio a alto, pero no tiene senadoras.

Durante el periodo de 1997-2000, como ya se dijo, nuevamente hubo un retroceso, pero éste es el más fuerte en la cámara de senadores ya que la presencia de 9 mujeres es apenas del $7 \%$ el porcentaje, más bajo. (ver mapa 4).

Además se observa una centralización de la presencia de mujeres, el Distrito Federal tuvo cuatro Senadoras, las cinco legisladoras de la cámara alta representaban a Chiapas, Jalisco, Tlaxcala, Yucatán y Zacatecas. Sin embargo coincide con un incremento en la cámara de diputadas ya que 14 entidades se establecen con una presencia baja y una más con una presencia media, Quintana Roo. Sólo tres entidades tenían representantes en ambas cámaras, el DF, presenta cuatro senadoras y un presencia baja de diputadas pero es la mejor posicionada, seguida de Tlaxcala y Zacatecas, ambas con una senadora y presencia baja de diputadas.

Ahora bien para el sexenio de 2000 a 2006, como ya se dijo, el PRI pierde por primera vez la presidencia de la República. Un dato importante es que en 2002 entraría en función la cláusula de género en donde no puede haber más del $70 \%$ de un género en la cámara, elemento que tampoco se cumple en esta legislatura, a pesar de un incremento en la presencia de mujeres en la Cámara Alta. (ver mapa 5 y 6).

Como se puede observar, en el mapa 5, en la Legislatura de 2000 a 2003 hay un incremento a 20 Senadoras, las entidades con representantes en la cámara alta son: Campeche, Chiapas, Distrito Federal con cuatro Senadoras, Estado de México, Guerrero, Michoacán, Nayarit, Querétaro, Quintana Roo, Sinaloa, Tabasco, Tlaxcala y Yucatán, y con dos senadoras Tamaulipas y San Luis Potosí. Al mismo tiempo en la Cámara de diputados se mantiene de 
Mujeres en el Legislativo Federal, una Mirada Espacial

México 1988-2011

Mapa 4. Diputadas y Senadoras en la LVII Legislatura, 1997-2000
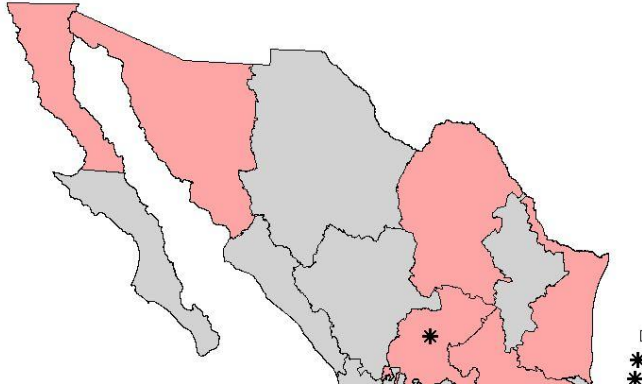

D.F.

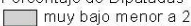

muy bajo menor a 20
matas

bajo 20 a 39

alto 60 a 79

muy alto igual o mayor a 80

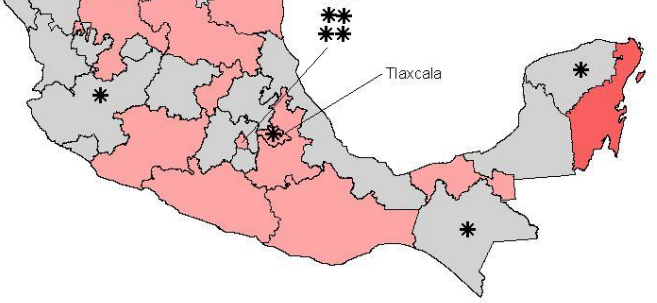

Senadoras

Elaboración propia
H. Cámara de Diputados

Album fotográfico Cámara de Diputados LVII Le gislatura

México 1998

$200000 \quad 0 \quad 200000400000$ Kilómetros

Archivo histórico y Memoria Legis lativa del Senado de la República

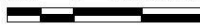

Mapa 5. Diputadas y Senadoras en la LVIII Legislatura, 2000-2003
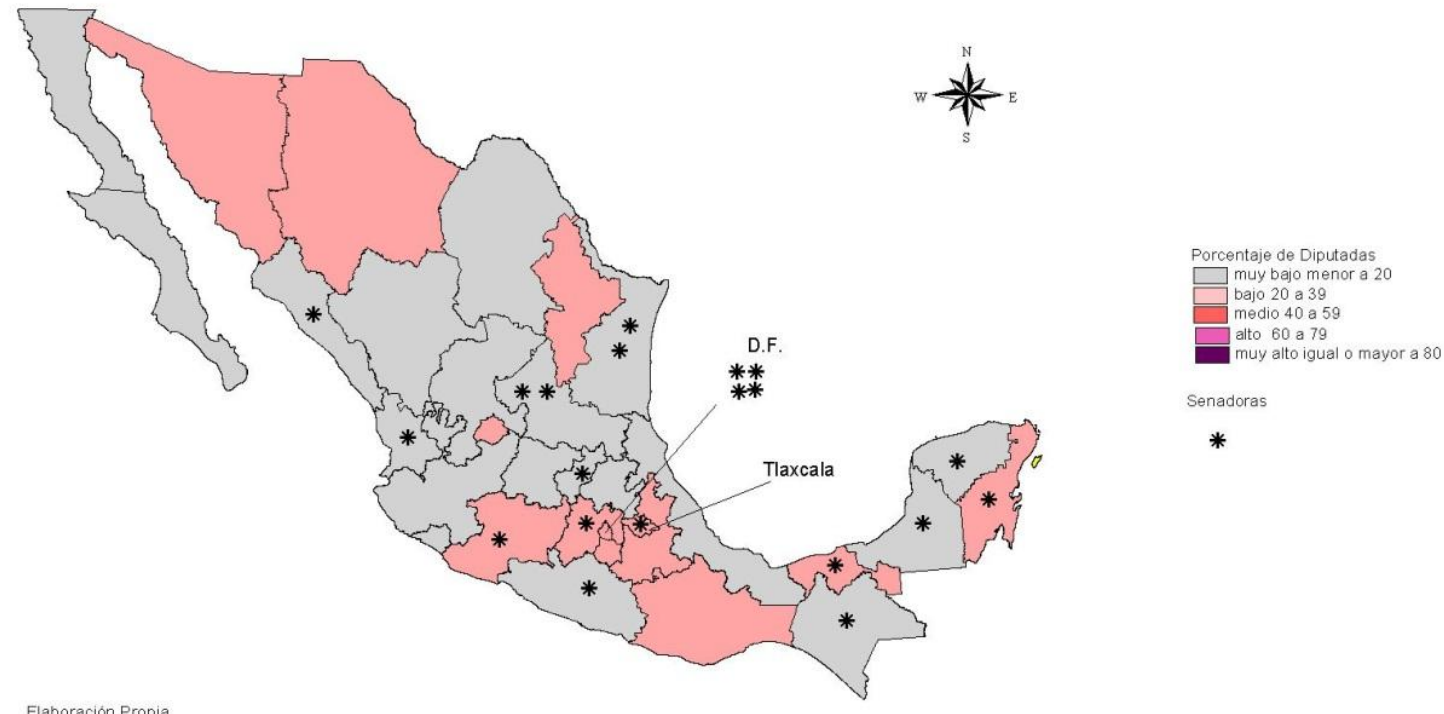

Fuente Cámara de Diputados

hmww.cddhcu gob. $m \times /$ servddd. México. 2006

H. Senado de la República M

$200000 \quad 0 \quad 200000400000$ Kilómetros 
manera similar en comparación a la anterior legislatura, en términos generales 13 entidades se encuentran en el rango de bajo.

Como podemos observar existe entidades en donde coinciden senadoras y diputadas, como el DF, que destaca por sus cuatro senadoras, seguida de Estado de México, Michoacán, Quintana Roo, Tabasco y Tlaxcala con una representante en la Cámara alta y con un rango bajo en la Cámara de Diputados. Es de llamar la atención que Tamaulipas y San Luis Potosí presentan a dos senadoras cada una, pero en diputaciones están en el rango de muy bajo.
En las elecciones intermedias de 2003, no se dan cambios en la Cámara alta, pero si en la de diputados y en ella se observa un avance general ya que, se presentan más entidades en un rango medio; Nuevo León, Aguascalientes, Yucatán y Campeche, quince entidades en un rango bajo. Por primera vez más de la mitad de las entidades llega a representación baja y media. Así aún cuando queda similar el número de senadoras existe un aumento de mujeres en el legislativo Federal. (ver mapa 6).

En la legislatura 2006 al 2009 se alcanzó el máximo en representación de mujeres en el legislativo

\section{Mapa 6. Diputadas y Senadoras en la LIX Legislatura, 2003-2006}

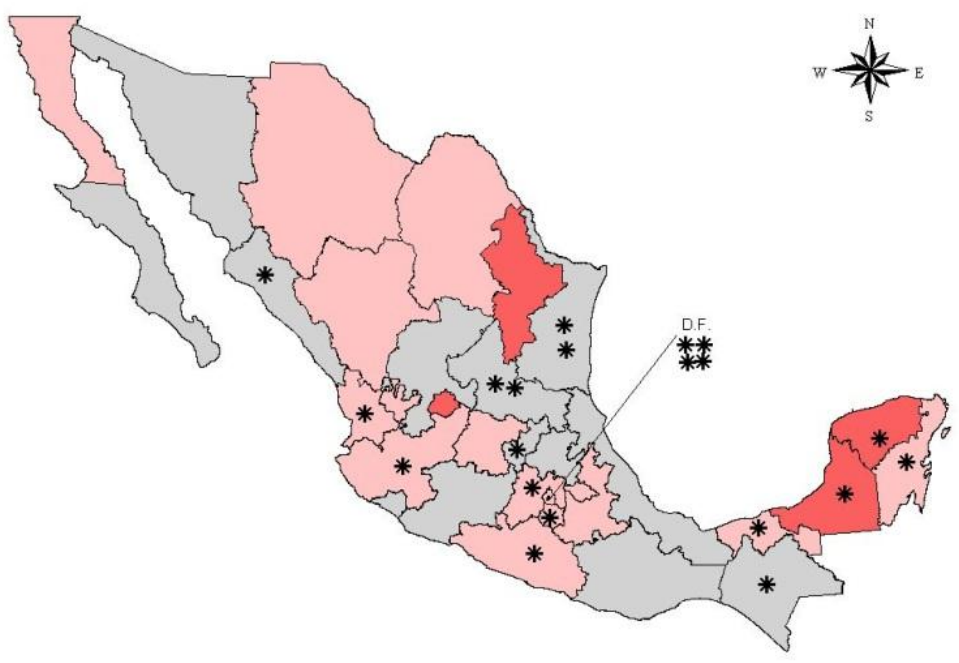

$200000 \quad 0 \quad 200000400000$ Kilómetros

Elaboracior Integración de Comisiones LIX Legislatur México. 2006
Archivo históricos Archivo historico y Memoria Legislativa del Senado de la República. México D.F. 2011 federal, oficialmente 31 Senadoras llegaron a la cámara alta, sin embargo, dos renunciaron en menos de tres meses, ambas del partido Verde Ecologista de México. Las entidades que tienen representantes mujeres en la cámara alta son: Distrito Federal con cuatro Senadoras, seguida de Sinaloa con tres representantes, Estado de México, Tamaulipas, Tlaxcala y Yucatán, con dos legisladoras cada una y con una representante en Aguascalientes, Baja
California Sur, Campeche, Colima, Chiapas, Chihuahua, Nuevo León, Michoacán, Puebla, Querétaro, Quintana Roo, Sonora, Tabasco, y Zacatecas. Y aunque aparece Jalisco, este no fue considerado ya la senadora que estaría por seis años, estuvo por siete días. (ver mapa 7). 




A pesar del incremento de mujeres en la legislatura no se alcanza el $30 \%$ establecido en la ley. Por entidad federativa existen avances interesantes de dejar constancia tal es el caso de Quintana Roo que tiene una senadora por entidad y presencia muy alta de diputadas, y Campeche con un rango alto de diputadas y con una Senadora, también destaca el Distrito Federal con cuatro senadoras, Sinaloa con tres curules en la Cámara alta ocupada por mujeres y el Estado de México, Tamaulipas y Tlaxcala con dos senadoras cada una de estas entidades, por su parte, Aguascalientes tienen un rango medio de Diputadas y una Senadora. Se identifica una presencia constante de entidades con Diputadas y Senadoras, al mismo tiempo que evidencia aquellas entidades que carecen de Senadoras y el rango de diputadas es muy bajo tal es el caso de Baja California, Coahuila, Guerrero e Hidalgo. Si bien no existen elecciones para senadoras en la jornada electoral intermedia para el cambio de diputados, si se observan transformaciones en la composición de esta cámara, ya que aunque se ha comentado que fueron treinta y una las senadoras, no obstante dos senadoras pidieron licencia, la primera seis días después de tomar posesión del cargo, María Irma Ortega Fajardo, ella es originaria del Estado de Jalisco y tomo posesión el 29 de septiembre y renuncio el 5 de Octubre de 2006. La segunda fue Gabriela Aguilar García quien pidió licencia el 28 de noviembre de 2006.

Caso contrario se registró en Yucatán donde la Senadora Ivonne Ortega Pacheco dejó el cargo dos meses después para contender por la gubernatura del estado, misma que ganó y se convirtió así en la segunda gobernadora durante el Siglo XXI.

Finalmente podemos observar en el mapa 8, que si bien no se mantiene el número de senadoras que supuestamente deberían de llegar a la cámara alta, el número de diputadas incrementan a su mayor nivel con 131 diputadas, poco a poco las entidades se alejan del rango muy bajo para mantenerse al bajo mayoritariamente, cinco presentan un rango medio: Coahuila; Tamaulipas, Aguascalientes, Hidalgo y Colima; Tlaxcala entra al alto y Nayarit se coloca en muy alto. (ver mapa 8). 


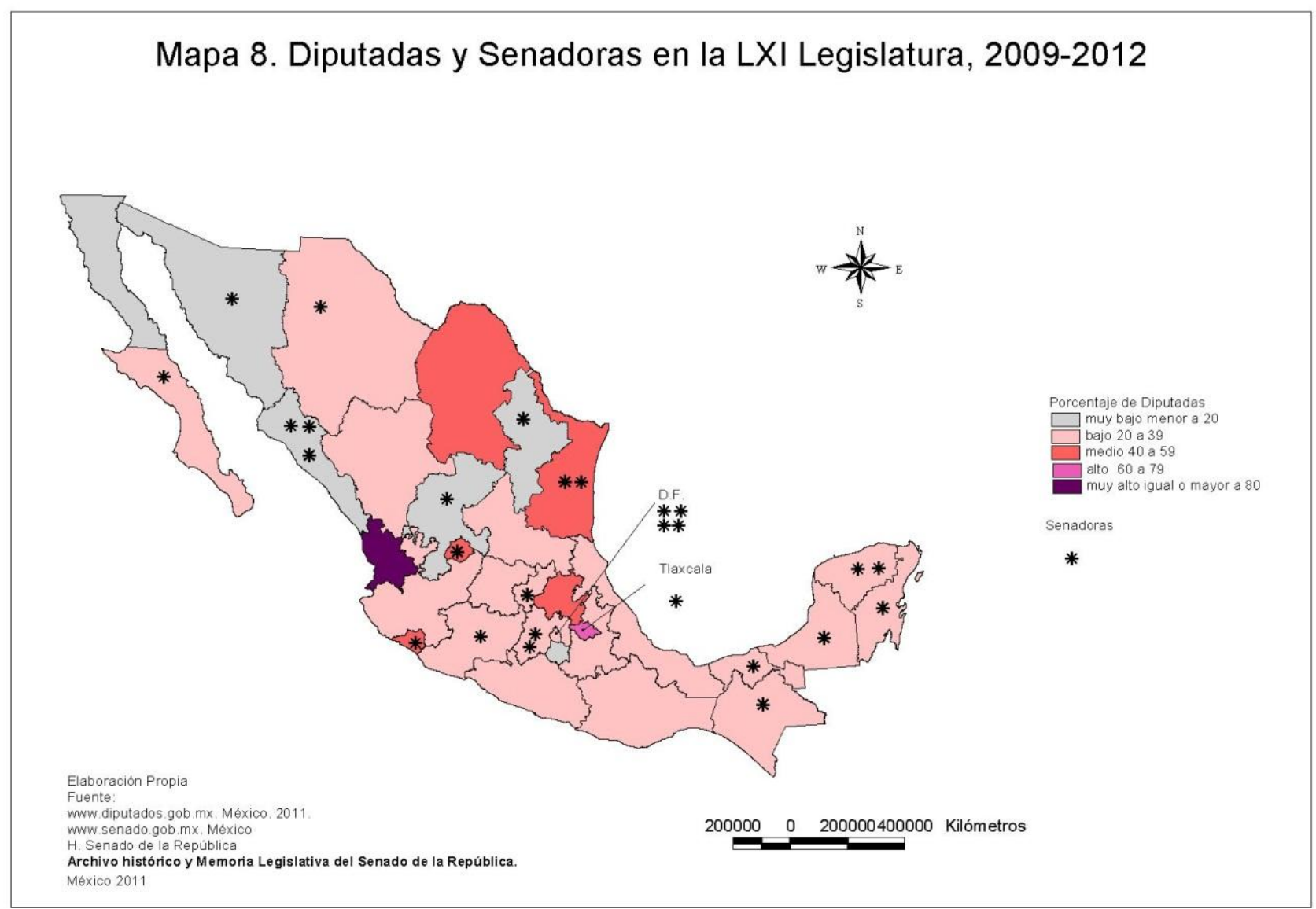

\section{Resultados}

Podemos observar un incremento constante en el número de mujeres en el Legislativo Federal, sin embargo este crecimiento no alcanza para dar cumplimiento a los tratados internacionales firmados por el estado Mexicano en equidad de Género, ni dar cabal cumplimiento a las normas establecidas $y$ signadas por el mismo Legislativo Federal, tampoco del Instituto Federal Electoral.

Identificamos a entidades con una presencia sostenida de Legisladoras de ambas cámaras, sin embargo existen entidades que durante el periodo de este estudio no presentan ninguna senadora tal es el caso de Veracruz y Guanajuato.

Con base en este estudio podemos observar que algunas entidades en las que las mujeres tienen mayores posibilidades de acceder a ser parte del poder legislativo, tal es el caso del Distrito Federal, el Estado de México, Querétaro, Tamaulipas, Yucatán, Tlaxcala y Quintana Roo.

Ahora bien esta escasa presencia de mujeres en el poder legislativo es parte de un círculo vicioso, ya que el que haya menos mujeres implica menos posibilidad de legislar con perspectiva de género, ahora bien tampoco es garantía de que al arribar más mujeres a las legislaturas esto vaya a suceder pero al menos existe una mayor posibilidad de que así sea.

Este es un primer acercamiento espacial a la incorporación de las mujeres al poder legislativo federal, sin embargo hacen falta estudios en diferentes escalas que nos permitan identificar los avances, inercias o retrocesos en una incorporación de las mujeres en situación de igualdad a las estructuras de poder, como un derecho histórico-geográfico demandado por mujeres y hombres.

\section{Referências}

BARRERA Bassols, Dalia. Participación Política de las Mujeres y gobiernos locales en México. México D.F.: GIMTRAP, 2002.

BARRERA, Bassols Dalia. Mujeres y gobiernos municipales en México. Cuicuilco Nueva Época, v. 6, n. 17, p. $87-99,1999$. 
BARRERA, Bassols Dalia. Mujeres, ciudadanía y poder. México. D.F.: Colegio de México, 2000.

BARRERA, Bassols Dalia.Mujeres que gobiernan municipios un perfil". En: BARRERA, Bassols Dalia; MASSOLO, Alejandra. Mujeres que gobiernan Municipios - Experiencias, aportes y retos. México: D.F. Colegio de México, 1998, p. 91-112.

FERNÁNDEZ PONCELA, Anna Ma. La política, la sociedad y las mujeres. México D.F.: Instituto Nacional de las mujeres, 2003.

FERNÁNDEZ PONCELA, Anna Ma. Las mujeres y la política: encuentros, desencuentros y perspectivas". En: FERNÁNDEZ PONCELA, Anna Ma. (Comp.). Participación política de las mujeres en México al final del Milenio. México D.F.: El Colegio de México, 1995, p. 11-19.

FERNÁNDEZ PONCELA, Anna Ma. Participación social y política de las mujeres en México: un estado de la cuestión. FERNÁNDEZ PONCELA, Anna Ma. (Comp.). Participación política de las mujeres en México al final del Milenio. México D.F.: El Colegio de México, 1995, p. 23-84.

FERNÁNDEZ, Paulina. Participación Política de la Mujer en México. En: FERNÁNDEZ PONCELA, Anna Ma. (Comp.). Participación política de las mujeres en México al final del Milenio. México D.F.: El Colegio de México, 1995, p. 85-106.

GONZÁLEZ, Philips Graciela. Género Femenino y Política: hacia la visibilidad de las mujeres del Partido de la Revolución Democrática. 2002. Tesis (Maestra en Antropología Social) Escuela Nacional de Antropología e Historia, México D.F.

HIDALGO, Antonieta. Mujeres Príistas Destacadas. Algunas estrategias de Ascenso y Legitimidad Política. En: BARRERA, Bassols Dalia. Mujeres Ciudadanía y Poder. Ciudad de México: El Colegio de México, 2000, p. 295-342.

HUERTA, Magdalena; MAGAR, Meurs Eric (Coord.). Mujeres Legisladoras en México: avances, obstáculos, consecuencias y propuestas. México D.F.: ITAM, 2006.

MASSOLO, Alejandra. Gobierno municipal y mujeres: un encuentro posible. En: BARRERA BASSOLS, Dalia; MASSOLO, Alejandra. Mujeres que gobiernan Municipios Experiencias, aportes y retos. México. D.F.: Colegio de México, 1998, p. 1327

MASSOLO, Alejandra. Participación femenina en el gobierno municipal. En: FERNÁNDEZ, Poncela Anna. Participación política de las mujeres en México al final del Milenio. México D.F.: El Colegio de México, 1995, p. 137-146.

MASSOLO, Alejandra. Pluralidad Política y pluralidad de género en favor de ayuntamientos democráticos. En: BARRERA BASSOLS, Dalia; MASSOLO, Alejandra. Mujeres que gobiernan Municipios Experiencias, aportes y retos. México D.F.: Colegio de México, 1998, p. 31-48.

Presidencia de la República. Directorio del Gobierno Mexicano. Presidencia de la República-Diana. 1992.

RAMIREZ, Flor. Directorio del Gobierno Mexicano. Presidencia de la República: Diana, 1989.

RODRIGUEZ, Beatriz. Mujeres y Participación Ciudadana en un ayuntamiento panista: Córdoba Veracruz. En: BARRERA BASSOLS, Dalia. Mujeres Ciudadanía y Poder. Ciudad de México: El Colegio de México, 2000, p. 227-293.

SAM, Magdalena. Mujeres gobernando en Veracruz: tres estudios de caso. En: BARRERA BASSOLS, Dalia; MASSOLO, Alejandra. Mujeres que gobiernan Municipios Experiencias, aportes y retos. México: Colegio de México, 1998, p. 63-82.

SAM, Magdalena. Mujeres Gobernantes: Los casos de Banderilla, Coatepec y Gutiérrez Zamora, Veracruz. En: BARRERA BASSOLS, Dalia. Mujeres, Ciudadanía y Poder. México: Colegio de México. 2000, p. $12-27$.

TUÑON, Enriqueta. ¡Por fin ...ya podemos elegir y ser electas! El sufragio femenino en México, 19351953. México D.F.: Plaza y Valdés-CONACULTAINAH. 2002.

ZARAGOZA, Juan Manuel. Relaciones de género en los gobiernos municipales de Morelos. En: BARRERA BASSOLS, Dalia; MASSOLO, Alejandra. Mujeres que gobiernan municipios: experiencias, aportes y retos. México: Colegio de México, 1998, p. $91-89$.

Archivo histórico y memoria legislativa - En: 
www.senado.gob.mx/servddd.http://www.senado.gob. $\mathrm{mx} /$ index.php?ver=sp\&mn=6; http://www.senado.gob. $\mathrm{mx} /$ index.php? $\mathrm{ver}=\mathrm{sp} \& \mathrm{mn}=6 \& \mathrm{sm}=$ sabiasque. Acceso julio de 2011 .

H. Senado de la República. Archivo histórico y Memoria Legislativa del Senado de la República. México. D.F., 2011.

H. Cámara de Diputados. Los Diputados de la nación III. Índice por Legislatura, H. Cámara de Diputados. México D.F., 1994.

H. Cámara de Diputados. Directorio oficial LV Legislatura 1996-1997. H. Cámara de Diputados. México. D.F. ,1994.

H. Cámara de Diputados. Álbum fotográfico. Cámara de Diputados. LVII Legislatura. México D.F., 1999.

H. Cámara de Diputados. Diputados Federales 20062009. Tercer año de ejercicio constitucional. LX Legislatura, México, 2009.

H. Cámara de Diputados. LX Legislatura. Diputados Federales. Primer año del ejercicio constitucional. México D.F., 2007. 